\title{
KLEIN GORDON FIELD IN FLRW SPACE-TIME
}

\author{
S. K. Sharma*, P. R. Dhungel** and U. Khanal*** \\ *B.P. Koirala Memorial Planetarium, Observatory and Science Museum Development Board, MoEST, Kathmandu, \\ Nepal. \\ **St. Xavier's College, Maitighar, Kathmandu, Nepal. \\ ***Central Department of Physics, Tribhuvan University, Kathmandu, Nepal.
}

\begin{abstract}
As a continuation of solving the equations governing the perturbation of the Friedmann-Lemaitre-RobertsonWalker (FLRW) space-time in Newman-Penrose formalism, the behaviour of the massive Klein-Gordon (KG) field coupled to the FLRW has been investigated. The Equation of Motion has been written and solved separately for radial and temporal parts. The former solution has come to be in terms of the Gegenbauer polynomials and spherical harmonics and the latter being in the WKB approximation. The particle current, energy momentum tensor and potential have also been obtained.
\end{abstract}

Keywords: FLRW spacetime; Perturbation; NP formalism; Klein-Gordon field; Jacobi polynomials.

\section{INTRODUCTION}

In this work, we investigate the behaviour of the massive Klein-Gordon $(\mathrm{KG})^{1,2}$ field coupled to the FriedmanLemaitre-Robertson-Walker (FLRW) background geometry. In our previous work $^{3}$, we were able to write down and solve the equations governing the perturbation of FLRW space-time in Neumann-Penrose formalism ${ }^{4,5}$. The angular eigen functions turn out to be the spinweighted spherical harmonics $p_{l}^{m}$ of weight $p=0, \pm 1, \pm 2$, corresponding to scalar, vectorial and tensorial perturbations. In the process, we have also been able to identify these as spherical harmonics formed with the appropriately weighted Jacobi polynomials $P_{p}^{(\alpha, \beta)}(\cos \Theta)$. Similarly, the temporal and radial part could be written in terms of the appropriate boostweighted functions. It was found that the equations governing the Maxwellian field in FLRW spacetime $^{6}$ were the same as that for the vectorial perturbation. The possibility that the spin-1 electromagnetic field could be the source of the rotational perturbations must be investigated in detail.

It was also found that the equations governing the scalar perturbation of the FLRW spacetime, density and pressure in particular, is the same as the conformally coupled massless KG field. This has motivated us to investigate the behaviour of the KG field as well. We had also worked on the Dirac field ${ }^{6}, 7$, and were under the impression that particular aspects of KG field would be duplicated there. But now we find that there are some specific differences. In particular, it was found that the mass of the Dirac field directly couples to the expansion rate of the Universe. In the case of the KG field, we will put in the coupling by hand. In the next Section, we set up the problem and solve the equation of motion. In Section III we discuss the energy-momentum of the field. The final Section contains some further consequences, and makes some concluding remarks.

\section{Klein Gordon Field}

The action integral in this case is given by,

$$
A=\int\left(\partial_{\mu} \Psi^{*} \partial^{\mu} \Psi-M^{2} \Psi^{*} \Psi-\xi R \Psi^{*} \Psi\right) \sqrt{g d}{ }^{4} x
$$

where the coupling constant $\xi$ can have any arbitrary value (but is equal to $1 / 6$ for conformal coupling), $\mathrm{g}=-\operatorname{detg}_{\mu \nu}$, and $\mathrm{R}=\frac{6}{\mathrm{a}^{3}}(\mathrm{a}=\mathrm{Ka})=8 \pi \mathrm{G}(\mathrm{Q}-3 \mathrm{P})$ is the trace of the Einstein equations in FLRW spacetime, prime denoting derivative with respect to the conformal time $\eta$. We may regard,

$V=\left[M^{2}+\xi R\right]|\Psi|^{2}$

as the potential. We use the metric $d s^{2}=a^{2}\left[d \eta^{2}-d r^{2}-S^{2}\left(d \Theta^{2}+\sin ^{2} \Theta d \varphi^{2}\right)\right]$ where $a$ is the scale factor and

$S=\frac{\sin \sqrt{k} r}{\sqrt{k}}\left\{\begin{array}{cl}\sin r, & k=1 \\ r, & k=0 \\ \sinh r, & k=-1\end{array}\right.$

respectively for the closed, flat and open models.

The Equation Of Motion (EOM), obtained by setting the variational derivative $\frac{\delta A}{\delta \Psi^{*}}=0$, is,

$\left[D_{\mu} D^{\mu}+M^{2}+\xi R\right] \Psi=0$.As $R$ depends only on $\eta$, the spatial and time part can be separated as $\Psi(\eta, r)=$ $T(\eta) f(r)$, whence we can write

$\frac{1}{a T} \frac{\partial}{\partial \eta}\left(\frac{\partial a T}{\partial \eta}\right)+M^{2} a^{2}+\left(\xi-\frac{1}{6}\right) a^{2} R=\frac{1}{S f} \nabla^{2} S f=-p^{2}$,

where the spatial gradient

$\nabla^{2}=\frac{1}{S^{2}} \frac{\partial}{\partial r} S^{2} \frac{\partial}{\partial r}+\frac{1}{S^{2} \sin \Theta} \frac{\partial}{\partial \Theta} \sin \Theta \frac{\partial}{\partial \Theta}+\frac{1}{S^{2} \sin ^{2} \Theta} \frac{\partial^{2}}{\partial \varphi^{2}}$

and $p^{2}$ is the separation constant that can be identified with the co-moving momentum; in this form, the essence of conformal coupling, $\xi=1 / 6$ becomes clear. The spatial part has the solution,

Author for Correspondence : S.K. Sharma, B.P. Koirala Memorial Planetarium, Observatory and Science Museum Development Board, Kathmandu, Nepal. Email: sksharmaktm@gmail.com 
$f(r)=N \sin ^{l}(\sqrt{k} r) C_{\frac{p}{\sqrt{k}}-l-1}^{(l+1)} \cos (\sqrt{k} r) Y_{l}^{m}(\theta, \phi)$, where $C_{n}^{(\alpha)}$ is the Gegenbauer polynomial ${ }^{10}$ and $Y$ is spherical harmonic $^{11}$. In the rest of this work, we take $K=1$, as $K=0$ and -1 can be recovered from the analytic continuation into the complex $r$-plane, with closed Universe on the real axis, open on the imaginary, and flat near the origin. This gives a more complete picture, as the near Universe must be flat, while the distant over-density regions evolve as closed and the under-density as open. 2

$f(\mathbf{r})$ is normalized with $\int|f(\mathbf{r})| \sin ^{2} r \sin \Theta d r d \Theta d \varphi=1$. Some modes of the radial solution are displayed in Fig. (1).

The temporal part, governed by,

$$
\left[\frac{\partial^{2}}{\partial \eta^{2}}+M^{2} a^{2}+p^{2}+\left(\xi-\frac{1}{6}\right) a^{2} R\right] a T=0,
$$

can be written as a series solution for any $a$ that is an analytic function of $\eta$. Another, particularly illustrative, solution can be represented in the WKB approximation ${ }^{12}$. We substitute a trial function $a T=\frac{1}{\sqrt{2}} e^{-i \int^{\eta} a \varepsilon d \eta^{\prime}}$, with $a \varepsilon=\sum a \varepsilon_{k} \delta^{k}$, replace $\eta \rightarrow \eta / \delta$ and equate equal powers of $\delta$ to end up with the recurrence relations,

$$
\begin{aligned}
& \left(a \varepsilon_{0}\right)^{2}=p^{2}+M^{2} a^{2}+(6 \xi-1) K, \\
& a \varepsilon_{1}=-i\left(\ln \sqrt{a \varepsilon_{0}}\right), \\
& 2, \\
& 2 a \varepsilon_{0} a \varepsilon_{2}+\left(a \varepsilon_{1}\right)^{+i}\left(a \varepsilon_{1}\right)=(6 \xi-1) \frac{a^{\prime \prime}}{a} \\
& \sum_{m=0}^{k} a \varepsilon_{k-m} a \varepsilon_{m}=-i\left(a \varepsilon_{k-1}\right), k>2 \ldots \ldots
\end{aligned}
$$

The particle current is $j_{\mu}=i\left[\Psi^{*} \partial_{\mu} \Psi-\left(\partial_{\mu} \Psi^{*}\right) \Psi\right]$. In particular, with $j_{\eta}=n U_{\eta}$ where $n$ is the particle number density, we find that,

$$
n a^{3}=i a^{2}\left[T^{*} T^{\prime}-T^{* \prime} T\right]|f(\mathbf{r})|^{2}
$$

is independent of $\eta$ due to the constancy of the Wronskian $W\left[a T^{*}, a T\right]$ that follows from Eq. (5). We may integrate Eq. (7) over the spatial volume to find,

$N a^{3}=2 \pi^{2} n a^{3}=i a^{2}\left[T^{*} T^{\prime}-T^{* \prime} T\right]=$ constant,

which

can be set equal to unity without loss of generality; $2 \pi^{2}$ is the volume of the closed Universe. This is just the statement that the total co-moving particle number remains constant during the evolution of the Universe.

\section{Energy Momentum}

The energy momentum tensor $T_{\mu \nu}=\frac{2 \delta A}{\delta g^{\mu \nu}}$ as derived from the action integral (1) is,

$$
\begin{aligned}
& T_{\mu \nu}=D_{\mu} \Psi^{*} D_{\nu} \Psi+D_{\nu} \Psi^{*} D_{\mu} \Psi-g_{\mu \nu} D_{\alpha} \Psi^{*} D^{\alpha} \\
& +\left(\begin{array}{cc}
\left.M^{2} g_{\mu \nu}-2 \xi G_{\mu \nu}\right) \Psi^{*} \Psi & \ldots \ldots . . .(8)
\end{array}\right.
\end{aligned}
$$

where $D_{\mu}$ is the covariant derivative. Substituting from the EOM in the conservation equation $D_{\mu} T_{v}^{\mu}=0$ results in the expression,

$\left[\Gamma_{\alpha v}^{\lambda}-\Gamma_{v \alpha}^{\lambda}\right] g^{\mu \alpha}\left(D_{\mu} \Psi^{*} D_{\lambda} \Psi+D_{\lambda} \Psi^{*} D_{\mu} \Psi\right)-2 \xi R^{\mu}{ }_{v} D_{\mu}|\Psi|^{2}=0$

In a torsion free space, the first term vanishes by definition, and the second term to vanish requires $|\Psi|^{2}$ to be a constant. Or we may take the alternative view that torsion is generated due to the gravitational interaction of the scalar field. Deeper investigation of this aspect is required. The energy-momentum can also be modified as discussed in Ref. ${ }^{13}$.

The trace of the energy-momentum is,

$$
\begin{aligned}
& T_{\mu}^{\mu}=\frac{R_{\Psi}}{8 \pi G}=(\rho-3 P)=\left\langle\frac{n M^{2}}{\varepsilon}\right\rangle \\
& =-2 D_{\mu}\left[\Psi^{*} D^{\mu} \Psi\right]+2 M^{2} \Psi^{*} \Psi .
\end{aligned}
$$

Where $R_{\Psi}$ is contribution to curvature scalar by the $\Psi$ field; the EOM and some results of scalar particle kinamatics in FLRW space like $v^{2} a^{2}=\frac{p^{2}}{\varepsilon^{2}}$ and $\frac{v^{2} a^{2}}{1-v^{2}}=\frac{p^{2}}{M^{2}}$ have been used to write the last line, where the first term is a total divergence whose volume integral can be made to vanish at the boundary and may be neglected. Integrating Eq. (10) over the three volume and some rearrangements give us,

$|a T|^{2}=\frac{N a^{3}}{2 a \varepsilon}$.

This can be compared to establish,

$N a^{3}=2 \pi^{2} n a^{3}=i W\left[a T^{*}, a T\right]=2 a \varepsilon|a T|^{2}=1$.

Assuming Bose-Einstein distribution, and summing over the momenta, we have $\mathrm{na}^{3}=$ $\frac{1}{2 \pi^{2}} \sum_{l=0}^{\infty} \sum_{p=l+1}^{\infty} \sum_{m=-l}^{l} \frac{1}{e^{a \varepsilon_{-1}}}=\frac{1}{\pi^{2}} \sum_{p=0}^{\infty} \frac{p^{2}}{e^{a \varepsilon-1}}$, where the factor of 2 in the last equation can be taken to be the number of degenaracies, representing the particles and anti-particles. Thus, the number of particles within $p$ and $p+1$ is given by,

$\Delta n a^{3}=\frac{1}{\pi^{2}} \frac{p^{2}}{e^{a \varepsilon}-1}$.

Using the lowest order of the WKB approximation for $a \varepsilon$ given in Eq. (6), we have plotted $n a^{3}$ as a function of $M a$. 


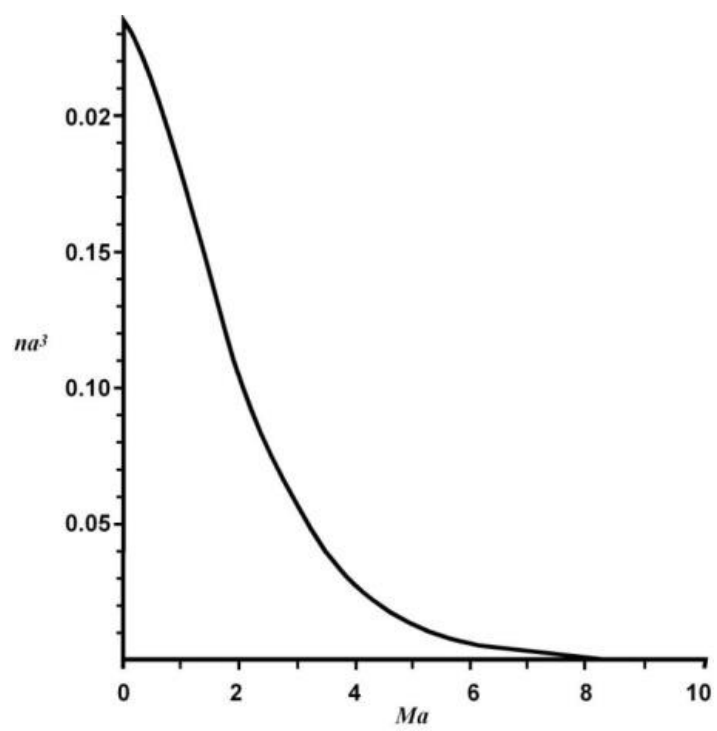

Fig 1: Relative particle number distribution as a function of $M a$. It is seen to decrease smoothly from the maximum value of $\mathbf{0 . 2 3 5}$ as $\mathrm{Ma}$ increases from $\mathbf{0}$.

The results of the discrete summation over $p$ of the distribution of Eq. (12) for closed space is very slightly less than the continuum limit to integration for flat space only for $M a<1$, becoming the same for greater values of $M a$. We stick to the discrete summation, and find the mean value for the trace of the energy momentum tensor given by,

$\langle\rho-3 P\rangle a^{4}=\left\langle n a^{3} \frac{M a^{2}}{a \varepsilon}\right\rangle=\frac{M a^{2}}{\pi^{2}} \sum_{p=0}^{\infty} \frac{p^{2}}{e^{a \varepsilon}-1}, \ldots$

which is plotted in Fig.(2). Its maximum value occurs at $M a=2.302$; at this value, maximum energy generates the minimum pressure. This must have some effect on structure formation, as this could be the most preferred state of matter, and the usual estimates of mass and pressure may not be correct. This could also account for some of the "missing matter" in the Universe.

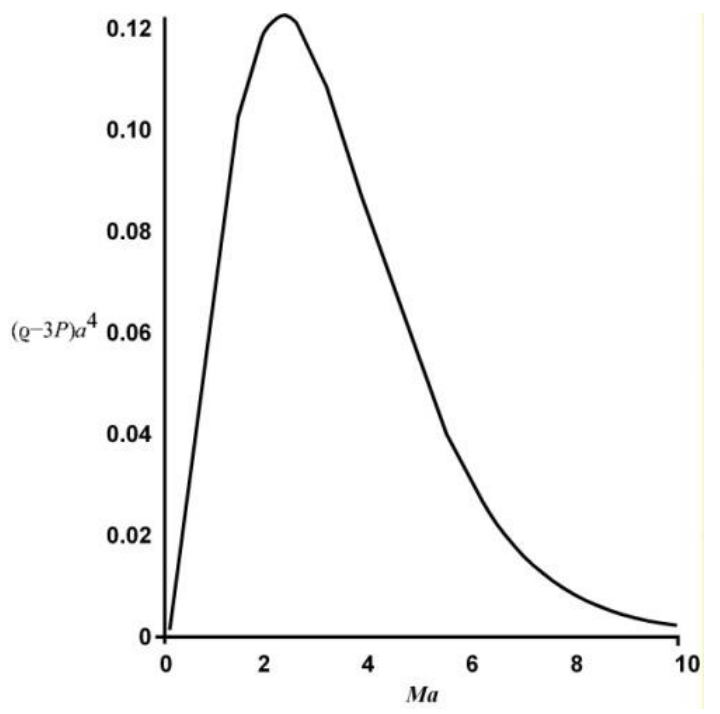

Fig 2: $\left(Q^{-3 P}\right) a^{4}$ as a function of $M a$. The maximum value of 0.1212 occurs at $M a=2.302$
Similarly, we find,

$T_{\eta \eta}=a^{2} \varrho=n a^{2} \varepsilon$

$=\frac{1}{a^{2}}\left(\Psi^{*} a^{2} \Psi^{\prime}\right)^{\prime}+\nabla_{i}\left(\Psi^{*} \nabla^{i} \Psi\right)$

$+2\left[a^{2} M^{2}+p^{2}-2 \xi\left(R_{\eta \eta}-a^{2} R\right)\right]|\Psi|$

Again, the first two terms in the last line can be made to vanish with appropriate boundary condition on integration, and we may write,

$a^{2} \varepsilon^{2}=p^{2}+a^{2} M^{2}+3 \xi\left(\frac{a^{\prime \prime}}{\mathrm{a}}+\frac{\mathrm{a}^{, 2}}{\mathrm{a}^{2}}+2 \mathrm{~K}\right)$,

where we can substitute from Friedman equation for the terms in the brackets. In the potential written in Eq.(2), if we use the $R_{\Psi}$ calculated in Eq. (10), we end up with a

$|\Psi|^{4}$ potential, $V=M^{2}|\Psi|^{2}+16 \pi G \xi M^{2}|\Psi|^{4}$, very naturally. This potential is itself not the one required for symmetry breaking. If we make the replacements $M^{2} \rightarrow-\mu^{2}$ and $\xi \rightarrow-\lambda$, as is usually done, then the it becomes the double-welled potential,

$\mathrm{V}=\mu^{2}|\Psi|^{2}+16 \pi G \lambda \mu^{2}|\Psi|^{4}$

required for spontaneous symmetry breaking as well as for inflation. Letting the field $\Psi=e^{i \chi} \psi$, it is seen that a vacuum expectation value of $\psi V_{a c}=\sqrt{32 \pi G \lambda}$ is developed.

\section{CONCLUSIONS}

We have solved the Klein-Gordon equation in the background gravitational field of the FLRW space time. Although the only scalar particle discovered to date is the Higgs, the $\mathrm{KG}$ field is used to model many physical phenomena like density and pressure perturbations, phase changes, etc. We find many interesting features of the solution. In particular, the trace of the energy-momentum tensor is found to achieve a maximum value when $M a=$ $2: 302$. Consequently, at this value, the field configures itself into a state of maximum energy density at minimum pressure. So the velocity dispersion in the over density regions of the Universe may not be what has been expected hitherto, and this may account for some of the dark matter.

When we feedback the trace of the energy-momentum of the $\mathrm{KG}$ field as a contribution to the curvature scalar, we find the potential to be of the $|\phi|^{4}$ form that is required for symmetry breaking. Thus, we end up naturally with a gravitational analog of Higgs mechanism. These are just some preliminaryresults, and we expect that further investigation of the behaviour of the $\mathrm{KG}$ field would reveal more important aspects of our Universe. 


\section{REFERENCES}

1. Peskin, M. E. and Schroeder, D. V. 1995. An Introduction to Quantum Field Theory. Westview Press, Colorado.

2. Redmond, P. J.1965. J. Math. Phys. 6: 1163.

3. Sharma, S. K. and Khanal, U. 2014. Int. J. Mod. Physics. D 23:1450017.

4. Newman, E. T. and Penrose, R. J. 1962. Math. Phys.3: 566.

5. Chandrasekhar, S. 1983. The Mathematical Theory of Black Holes Clarendon Press, Oxford.

6. Khanal, U. 2006. Class Quantum. Grav.23: 4353.

7. Dhungel, P. R. and Khanal, U. 2013. Chinese J. of Phys. 51: 882.
8. Kolb, E. W. and Turner, M. S. 1990. The Early Universe. AddisonWesley, New York.

9. Weinberg, S. Cosmology 2008 . Oxford Press, Oxford.

10. Gradshteyn , I. S. and Ryzhik, I. M. 1980. Table of Integral, Series and Products. Academic, New York.

11. Hobson, E. W. 1931. The Theory of Spherical and Ellipsoidal Harmonics. Chelsea, New York, 1931.

12. Landau, L. D. and Lifshitz, E. M. 1977. Quantum Mechanics. Nonrelativistic Theory. Pergamon Press, New York, 3rd edition.

13. Forger, M. and Romer, H. July 2003. Current and Energy Momentum in Classical Field Theory: A Fresh Look at an Old Problem. preprint, Universitat Freiburgh THEP 03/10. 